\section{Kidney Blood Pressure Research}

Kidney Blood Press Res 2018;43:616-627

DOI: $10.1159 / 000489146$

Published onlıne: 24 April, 2018

Accepted: 12 April, 2018

This article is licensed under the Creative Commons Attribution-NonCommercial-NoDerivatives 4.0 International License (CC BY-NC-ND) (http://www.kargercom/Services/OpenAccessLicense). Usage and distribution tional License (CC BY-NC-ND) (http://www.karger.com/Services/OpenAccessLicense). Usage and dist
for commercial purposes as well as any distribution of modified material requires written permission.

\title{
Nestin Improves Preeclampsia-Like Symptoms by Inhibiting Activity of Cyclin- Dependent Kinase 5
}

\author{
Xu Yang Yiling Ding Mengyuan Yang Ling Yu Yun Hu Yali Deng \\ Department of Obstetrics and Gynecology, the Second Xiangya Hospital, Central South University, \\ Changsha, Hunan, China
}

\section{Key Words}

Preeclampsia • Nestin • Podocyte $•$ Cyclin dependent kinase $•$ Glomerular filtration barrier

\begin{abstract}
Background/Aims: Preeclampsia (PE) is a pregnancy-specific hypertensive disorder that is characterised by a high incidence of hypertension and proteinuria. Podocytes are involved in the formation of a split membrane, which is the last barrier preventing the leakage of protein into the urine. Nestin, a cytoskeleton protein, is expressed stably in podocytes. However, the association between the Nestin concentration in urine and the progression of PE and the role of Nestin in PE remains unclear. Methods: In the present study, a mouse podocyte cell line, PE-like animal model and PE patients' urine samples were used. Eilsa kits were used to detect the levels of proteins expression in urine samples from patients and animal models. Western Blotting and immunofluorescence were used to detect proteins expression levels in cell samples and animal tissue samples. Flow cytometry was used to detect the level of apoptosis in cells. Tunel assay was used to detect the levels of apoptosis in animal tissue samples. Results: Nestin levels were significantly increased in PE patients than in hypertensive patients and healthy subjects, and positively correlated with proteinuria and podocalyxin. Ang II treatment decreased the expression of Nestin and Podocin in a time- and dose- dependent manner in podocytes. Restoration of the Nestin levels could reverse Ang II-induced F-actin degradation and attenuate Ang II-mediated podocyte apoptosis, while knockdown of the Nestin level exhibited the opposite. Moreover, the protective role of Nestin on podocytes is mediated by inhibition of the kinase activity of CDK5. In PE-like animal model induced by L-NAME injection, restoration of Nestin lowered the pressure and proteinuria concentration, attenuated the loss of podocytes, and decreased the expression of p35, p53 and the activity of CDK5 kinase, as compared with the control. Conclusions: Our findings suggest that Nestin could improve preeclampsia-like symptoms by inhibiting the activity of CDK5, and Nestin may become a new prognostic factor and a potential therapy target for PE.




\section{Kidney Blood Pressure Research}

Kidney Blood Press Res 2018;43:616-627

\begin{tabular}{l|l}
\hline DOI: $10.1159 / 000489146$ & (C) 2018 The Author(s). Published by S. Karger AG, Basel
\end{tabular}

Published onlıne: 24 April, 2018 www.karger.com/kb

Yang et al.: Nestin/CDK5 Improves Preeclampsia-Like Symptoms

\section{Introduction}

Hypertensive disorder-complicating pregnancy (HDCP), a pregnancy-specific disease responsible for $20 \%-25 \%$ of perinatal death, causes more than 50,000 maternal deaths every year across the world [1]. Preeclampsia (PE) is the high incidence of HDCP and is characterized by hypertension and proteinuria. It is often accompanied by edema, hyperuricemia and other clinical manifestations that can cause multiple organ dysfunction, intrauterine growth restriction, fetal stricken and postpartum haemorrhage [2]. Clinically, the level of proteinuria often indicates the severity of PE [3]. The glomerular filtration barrier is composed of capillary endothelial cells, glomerular basement membrane, and podocytes from the inside out of the filter barrier, which functions in maintaining the integrity of the membrane. Podocytes are located on the outermost layer of the glomerular filtration barrier, and are involved in the formation of the split membrane, which is the last barrier that challenges prevention of protein leakage into the urine [4]. Abnormalities of podocyte morphology, function, and number that are induced by several factors can cause podocyte damage, which can clinically resultin proteinuria [5]. Increased podocytes were observed in the urine samples from glomerular disease patients, along with the loss of papocytes in the glomeruli, which was closely related to proteinuria excretion [6]. These findings suggest that PE may cause podocyte damage, which in turn results in dysfunction of the glomerular filtration system, eventually leading to proteinuria.

Nestin, a cytoskeleton protein, is stably expressed in podocytes [7]. Nestin expression was also detected in normal and pre-eclamptic placental tissues and in endothelial cells of the chorionic villi [8]. It has been reported that the expression of Nestin in the podocytes is low in nephropathy with proteinuria [9-11]. However, the relationship between the concentration of Nestin in urine samples and the progression of PE or its role in PE remains unclear. The aim of this study is to evaluate the role and mechanism of Nestin in PE both in vitro and in vivo.

\section{Materials and Methods}

\section{Human urine sample collection}

Clinical data and urine samples were collected from 74 pregnant women in the Second Xiangya Hospital of Central South University during June-December 2016. The subjects were divided into three groups: healthy control, hypertensive and PE. The characteristics of the groups were: healthy control group $(n=15)$, pregnancy blood pressure $<140 / 90 \mathrm{mmHg}$ and no internal and obstetric complications; hypertensive group ( $n=17)$, high blood pressure before pregnancy, pregnancy blood pressure $>140 / 90 \mathrm{mmHg}$; PE group ( $\mathrm{n}=42)$, no hypertension before pregnancy, pregnancy blood pressure $>140 / 90 \mathrm{mmHg}$, proteinuria $>300 \mathrm{mg} / 24 \mathrm{~h}$ in urine. All of the above subjects were free of other infectious diseases, smoking, drinking and other bad habits.

The study was approved by the Ethics Committee of the Xiangya Second Hospital of Central South University. The subjects provided consent for urine sample collection. The urine samples were collected in sterile containers and centrifuged at $1500 \mathrm{rpm}, 4^{\circ} \mathrm{C}$ for $20 \mathrm{~min}$. The supernatants were collected and stored at $-70^{\circ} \mathrm{C}$ until further use.

Cell culture

Mouse podocytes were obtained from the BeNa Culture Collection (cat no. BNCC337685, Beijing, China). The cells were cultured as describedin a previous study [12]. The cells have a temperature-sensitive SV40 large T-cell antigen controlled by a $\gamma$-interferon-inducible $\mathrm{H}-2 \mathrm{~K}^{\mathrm{b}}$ promoter. To induce proliferation, cells were grown in collagen I-coated plastic culture bottles (Thermo Fisher, Waltham, MA, USA) at $33^{\circ} \mathrm{C}$ in RPMI 1640 medium supplemented with $10 \%$ foetal bovine serum (FBS, Gibco, Grand Island, NY, USA) and 10 U/ml $\gamma$-interferon (under growth-permissive conditions). To induce quiescence and differentiate thephenotypes, the podocytes were grown at $37^{\circ} \mathrm{C}$ without $\gamma$-interferon (under growth-restrictive conditions) in RPMI 1640 


\section{Kidney Blood Pressure Research}

Kidney Blood Press Res 2018;43:616-627

\begin{tabular}{l|l}
\hline DOI: 10.1159/000489146 & (C) 2018 The Author(s). Published by S. Karger AG, Basel \\
Published
\end{tabular}

Published onlıne: 24 April, 2018 www.karger.com/kbr

Yang et al.: Nestin/CDK5 Improves Preeclampsia-Like Symptoms

medium supplemented with 10\% FBS. All studies were performed on days 10-14 for cells grown under restrictive conditions. The medium was refreshed every 2 days. The cells were passaged in a 1:3 ratio when confluence reached $80 \%$.

\section{Cell treatment}

To mimic the hypertension condition in vitro, podocytes were treated with Ang II $(0.001,0.01,0.1,1$, $10 \mu \mathrm{M}$ ) for $24 \mathrm{~h}$ or with $1 \mu \mathrm{M}$ Ang II for $0,6,12,24$ and $48 \mathrm{~h}$. To inhibit the activity of CDK5 kinase, $0.2 \mu \mathrm{M}$ Roscovitine (cat no: S1153, Selleck, Shanghai, China) was added into the medium for $24 \mathrm{~h}$.

To knockdown Nestin levels, lentivirus containing Nestin siRNA (Genepharma, Shanghai, China) were constructed. To overexpress Nestin, an adenoviral vector PGMAV-P1 (Genomeditech, Shanghai, China) containing Nestin expression plasmid was constructed. To generate recombinant adenoviruses, HEK293 cells were co-transfected with the recombinant plasmid and the infectious genomic plasmid (pBHGE3i) using liposomes. Cells were plated in 6-well clusters or in 96-well plates and infected with the Nestinoverexpression-mediating adenovirus for $48 \mathrm{~h}$ for further assays or protein extraction.

\section{PE-like animal model preparation}

A total of 45 (15male, 30 female), 8 weeks old ICR mice were purchased from Shanghai SJA Experimental Co., Ltd. (Shanghai, China). After 4 weeks, the female and male mice (2: 1 ratio) were regularly placed in a cage overnight. The next day at around noon, the female mice were checked for the formation of a vaginal suppository, which suggested successful pregnancy; this day was recorded as the first 0.5 day of pregnancy (i.e., d 0.5). The pregnant animals were randomly divided into three groups: normal (control, $n=10$ ), PE model (PE, $n=10$ ) and PE plus Nestin overexpression (Nestin, $n=10)$. The PE mice were subcutaneously injected with L-NAME $\left(150 \mathrm{mg} \cdot \mathrm{kg}^{-1} \cdot \mathrm{d}^{-1}\right.$; Sigma-Aldrich, Santa Clara, CA, USA) on the back of the neck for 10 days from day 7.5 of gestation. For the overexpression of Nestin in PE mice, intravenous injection (approximately $10^{8} \mathrm{pfu}$ ) of Nestin overexpression adenovirus was performed in the mice via their tail veil at day 7.5 of gestation. The control group was injected with normal saline.

All animals were placed in metabolic cages and provided with free drinking water, and $24 \mathrm{~h}$ urine samples were collected. All urine specimens were treated with toluene $(1 \mathrm{ml}$ urine was added to $5 \mu \mathrm{l}$ toluene), and stored at $-80^{\circ} \mathrm{C}$. The tail artery systolic pressure was measured with an intelligent noninvasive sphygmomanometer (BP-2006A) in the quiet state of the fixed time point. Animals were sacrificed on day 18.5 of gestation. Both kidneys were removed. One kidney was placed in a cryopreserved tube and stored in liquid nitrogen. The other kidney was fixed in neutral formaldehyde to prepare paraffin sections for pathologic examination.

\section{Measurement of the concentration of proteinuria}

The Bradford Protein Assay Kit (cat no.P0006, Beyotime, Hangzhou, China) was used to detect the concentration of urinary protein in urine samples according to the manufacturer's instructions. Urine samples were diluted 1-fold with pre-cooled PBS. Diluted urine (8 $\mu \mathrm{l})$ and $12 \mu \mathrm{l}$ of pre-cooled PBS were added to a 96-well plate, to each well of which $200 \mu \mathrm{l}$ of the G250 staining solution was added. The wells were placed at room temperature for $5 \mathrm{~min}$ and the absorbance was measured immediately with a microplate reader at $595 \mathrm{~nm}$. The protein concentrations in the sample were calculated according to a standard curve.

\section{Measurement of Podocin and Nestin in urine samples}

ELISA kits (ELISA Kit for Podocin, cat no. SEA938Hu and ELISA Kit for Nestin (NES), cat no. SEA500Hu, CLOUD-CLONE CORP. Katy, TX, USA) were used to estimate the levels of podocyte marker Podocin and Nestin in urine samples according to the manufacturer's instructions. The final concentrations were calculated by multiplying the actual measured concentrations by the appropriate dilution factor.

\section{Phalloidin immunofluorescence}

The Phalloidin-iFluor 488 Reagent/CytoPainter (cat no. ab176753, Abcam, Cambridge, UK), one of a series of phalloidin conjugates that selectively binds to F-actin, was used to stain F-actin. The slide culture podocytes were washed with PBS (with $0.1 \%$ Tween) for 5 min and fixed with $4 \%$ paraformaldehyde at room temperature for $20 \mathrm{~min}$, followed by washing with PBS (with $0.1 \%$ Tween) thrice for 5 min each. After 


\section{Kidney Blood Pressure Research}

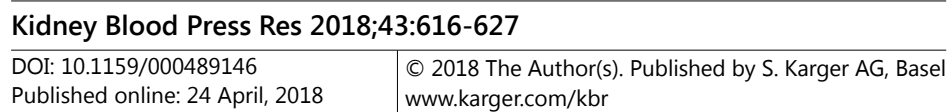

Yang et al.: Nestin/CDK5 Improves Preeclampsia-Like Symptoms

10 min of permeabilisation (with PBS 0.3\% Triton-X-100) and three washes washing with PBS (with $0.1 \%$ Tween) for $5 \mathrm{~min}$ each, the slides were stained with phalloidin for $1 \mathrm{~h}$ at room temperature in the dark. After another round of three washes with PBS (with $0.1 \%$ Tween) for 5 min each time, the slides were mounted with ProLong Gold Antifade Mountant containing DAPI on microscope slides dried overnight at $4^{\circ} \mathrm{C}$ and imaged on the following day.

\section{Podocin Immunofluorescence}

The slide culture podocytes were washed with PBS and fixed with $10 \%$ ethyl alcohol for $4 \mathrm{~h}$ at $4^{\circ} \mathrm{C}$. After blocking with $5 \%$ normal goat serum, the slides were incubated with the primary antibody Podocin (cat no. 20384-1-AP; Proteintech, Wuhan, China) overnight at $4^{\circ} \mathrm{C}$. The slices were then washed with TBST and incubated with Cy3 (cat no. SA012, Auragene, Changsha, China) for $2 \mathrm{~h}$ at room temperature. Finally, the slices were washed with TBST and counterstained with DAPI (1:1000 dilution; Sigma-Aldrich) for 5 min and then visualised under a microscope.

\section{Flow cytometric analysis of apoptosis}

After the indicated treatment, the cells were digested with trypsin without EDTA and washed twice with PBS. The cells were collected by centrifugation (at $2000 \mathrm{rpm}$ ) for $5 \mathrm{~min}$. The eBioscience ${ }^{\mathrm{TM}}$ Annexin V Apoptosis Detection Kit (cat no. 88-8102-72, Thermo Fisher Scientific, USA) was used to analyze for apoptosis, according to the manufacturer's instructions. Binding Buffer $(500 \mu \mathrm{l})$ was added to the suspended cells, Annexin V-FITC (5 $\mu \mathrm{l})$ was added and mixed well, and then propidium iodide ( $5 \mu \mathrm{l})$ was added and mixed well. After $10 \mathrm{~min}$ of reaction in the dark at room temperature, the cells were analysed within $1 \mathrm{~h}$ by flow cytometry.

\section{Western blotting}

After the indicated treatment, the cells were digested and collected. The protein contents were extracted from the cells with RIPA buffer. The BCA Protein Assay Kit (cat no. \#7780, Cell Signaling Technology, Danvers, MA, USA) was used to determine the protein concentration, according to the manufacturer's instructions. Protein $(60 \mu \mathrm{g})$ was added on to a 10\% SDS-PAGE gel, separated, and then transferred onto a nitrocellulose membrane (Boster, Wuhan, China). The membranes were incubated with primary antibody (anti-CDK5, 1:500, cat no. Ab40773, Abcam; anti-p35, 1:1000, cat no. Ab66064, Abcam; anti-p53, 1:500, cat no. Ab26, Abcam; anti-Podocin, 1:500, cat no. Ab181143, Abcam; anti-Nestin, 1:500, cat no. ab6142, Abcam, or anti- $\beta$-actin, 1:100, cat no. SC-365062, Santa Cruze) at $4^{\circ} \mathrm{C}$ overnight. Signals were visualised by using ECL Substrates (Millipore, MA, USA). The integrated optical density (IOD) for each band was analyzed by Gel pro 4.0 software.

\section{Measurement of CDK5 kinase activity}

After the indicated treatment, the cells were digested and collected. The protein content of the cells was extracted with RIPA buffer. About $10 \%$ of the lysate was used as input. Laemmli buffer $(5 \times, 1 \mathrm{ml}$ glycerol, $1.56 \mathrm{ml} 2 \mathrm{M}$ Tris- $\mathrm{HCl}, 2.5 \mathrm{ml} \beta$-mercaptoethanol and $1 \mathrm{ml} 0.2 \%$ bromophenol blue) was added to input to make the final concentration of the buffer to $1 \times$. The lysate was centrifuged at $10,000 \times g$ and $4^{\circ} \mathrm{C}$ for $10 \mathrm{~min}$, and the resulting supernatant was collected. Protein A Sepharose CL-4B (50 $\mu \mathrm{l}$, cat no. 17-078001 , GE Healthcare) was added to the supernatant, which was then placed on a shaker at slow speed at $4^{\circ} \mathrm{C}$ overnight. After $2 \mathrm{~min}$ of $3000 \times \mathrm{g}$ centrifugation at $4^{\circ} \mathrm{C}$, the supernatant was collected and analyzed by the BCA Protein Assay Kit (cat no. \#7780, Cell Signaling Technology, Danvers, MA, USA) in order to determine the protein concentration. Lysate $(500 \mu \mathrm{l})$ containing $1 \mathrm{mg}$ protein and $2 \mu \mathrm{g}$ CDK5 antibody (cat no. Ab40773, Abcam,) was added and mixed well. The mixture was incubated at $4^{\circ} \mathrm{C}$ for $3 \mathrm{~h}$. Then, Protein-A beads $(50 \mu \mathrm{l})$ were added to the mixture and incubated at $4^{\circ} \mathrm{C}$ for $2 \mathrm{~h}$. The mixture was centrifuged $\left(4^{\circ} \mathrm{C}\right.$, $3,000 \times g$ ) for 2 min to remove the supernatant. Lysis buffer $(500 \mu \mathrm{l}$ ) containing $0.1 \% \mathrm{NP}-40$ was added to the precipitate and incubated at $4^{\circ} \mathrm{C}$ for $10 \mathrm{~min}$ and then centrifuged $\left(4^{\circ} \mathrm{C}, 3,000 \times \mathrm{g}\right)$ for $2 \mathrm{~min}$ to remove the supernatant. Kinase buffer ( $500 \mu \mathrm{l}, 1 \mathrm{M}$ MOPS (pH=7.0), $1 \mathrm{M} \mathrm{MgCl}_{2}$, 0.5 M EDTA (pH8.0), 0.5 M EGTA (pH8.0)) without ATP or histone $\mathrm{H} 1$ was added to the precipitate and shaken for $10 \mathrm{~min}$ at $4^{\circ} \mathrm{C}$, followed by centrifugation $\left(4^{\circ} \mathrm{C}, 3,000 \times \mathrm{g}\right)$ for $2 \mathrm{~min}$ to remove the supernatant. Next, $25 \mu \mathrm{l}$ kinase buffer (containing $100 \mathrm{ng} / \mu \mathrm{l}$ of histone $\mathrm{H} 1$ and $25 \mu \mathrm{M} \mathrm{ATP})$ was added to resuspend the precipitate, and then $2 \mu \mathrm{l}\left[\gamma^{-32} \mathrm{P}\right]$ ATP 


\section{Kidney Blood Pressure Research}

working solution $\left(0.1 \mu \mathrm{Ci} / \mu \mathrm{l}\left[\gamma^{-32} \mathrm{P}\right]\right.$ ATP, $10 \mathrm{mM}$ ATP, $25 \mathrm{mM}$ magnesium acetate) was added and mixed well. The mixture was incubated for $30 \mathrm{~min}$ at $30^{\circ} \mathrm{C}$. Laemmli buffer $(5 \mu \mathrm{l}, 5 \times)$ was added to the mixture. Finally, the mixture conjunct with ${ }^{32} \mathrm{P}$ was loaded onto a $10 \%$ SDS-PAGE gel, separated, and then transferred onto a PVDF membrane (Boster, Wuhan, China). The resultantbands were visualised by autoradiography.

TUNEL assay

The TUNEL assay method was used to detect DNA breaks by using the One Step TUNEL Apoptosis Assay Kit (cat no. C1088, Beyotime, Hangzhou, China) according to the manufacturer's instructions. The cells were counterstained with 4', 6-diamidino-2-phenylindole (DAPI). Fluorescent images were acquired with the Leica DM IRB Epifluorescence Microscope.

\section{Statistical analysis}

The SPSS Statistics 17.0 softwarepackage was used for dataanalysis. All data from 3 independent experiments were expressed as the mean \pm SD. Student's $t$ test was used for the statistical analysis of data obtained from two groups, and data from more than two groups were analyzed by one-way ANOVA followed by Tukey's multiple comparison test. Differences resulting in $P<0.05$ were considered statistically significant.

\section{Results}

The association of Nestin levels and podocyte cell marker Podocin in urine samples from PE patients

To investigate the role of Nestin in PE, we first detected the levels of Nestin in urine samples obtained from PE patients. Urine samples were collected from health pregnant women (control), hypertensive patients and PE patients. We confirmed that the concentration of proteinuria in PE patients was significantly higher than those in health controls and hypertensive patients (Fig. 1A). In addtion, podocyte cell marker podocalyxin levels were significantly increased in hypertensive patients and PE patients as compared to that in health controls, while podocalyxin levels were higher in PE patients than in hypertensive patients (Fig. 1B). Morever, Nestin levels were significantly increased in PE patients than in hypertensive patients or health controls (Fig. 1C). Furthermore, we analysed the correlation between Nestin, preoteinuria and podocalyxin in PE patients. The results showed that proteinuria levels were positively correlated with the podocalyxin level $\left(\mathrm{R}^{2}=0.7246, P<0.01\right.$; Fig. 1D), and Nestin levels were positively correlated with proteinuria and podocalyxin $\left(\mathrm{R}^{2}=0.7216, P<0.01\right.$, Fig. 1E; $\mathrm{R}^{2}=0.7147, P<0.01$, Fig. $\left.1 \mathrm{~F}\right)$. The increased podocalyxin and Nestin levels in urine samples from PE patients suggest damage of the podocytes in kidney.

\section{Ang II reduced Nestin expression and induced apoptosis in podocyte}

To investigate whether hypertension can lead to the down-regulation of Nestin, we treated podocytes with Ang II in vitro. Ang II treatment was found to decrease the expression of Nestin and Podocin in a time- and dose-dependent manner (Fig. 2A). Next, we treated the cells with $1 \mu \mathrm{m}$ Ang II for 24 and $48 \mathrm{~h}$ and found that Ang II treatment induced F-actin degradation and down-regulation of Podocin in a time-dependent manner (Fig. 2B). In addition, Ang II treatment led to an increase in podocyte apoptosis (Fig. 2C). Furthermore, Ang II treatment increased the expression of p35 and p53, while it did not alter the expression of CDK5 (Fig. 2D). 


\section{Kidney Blood Pressure Research}

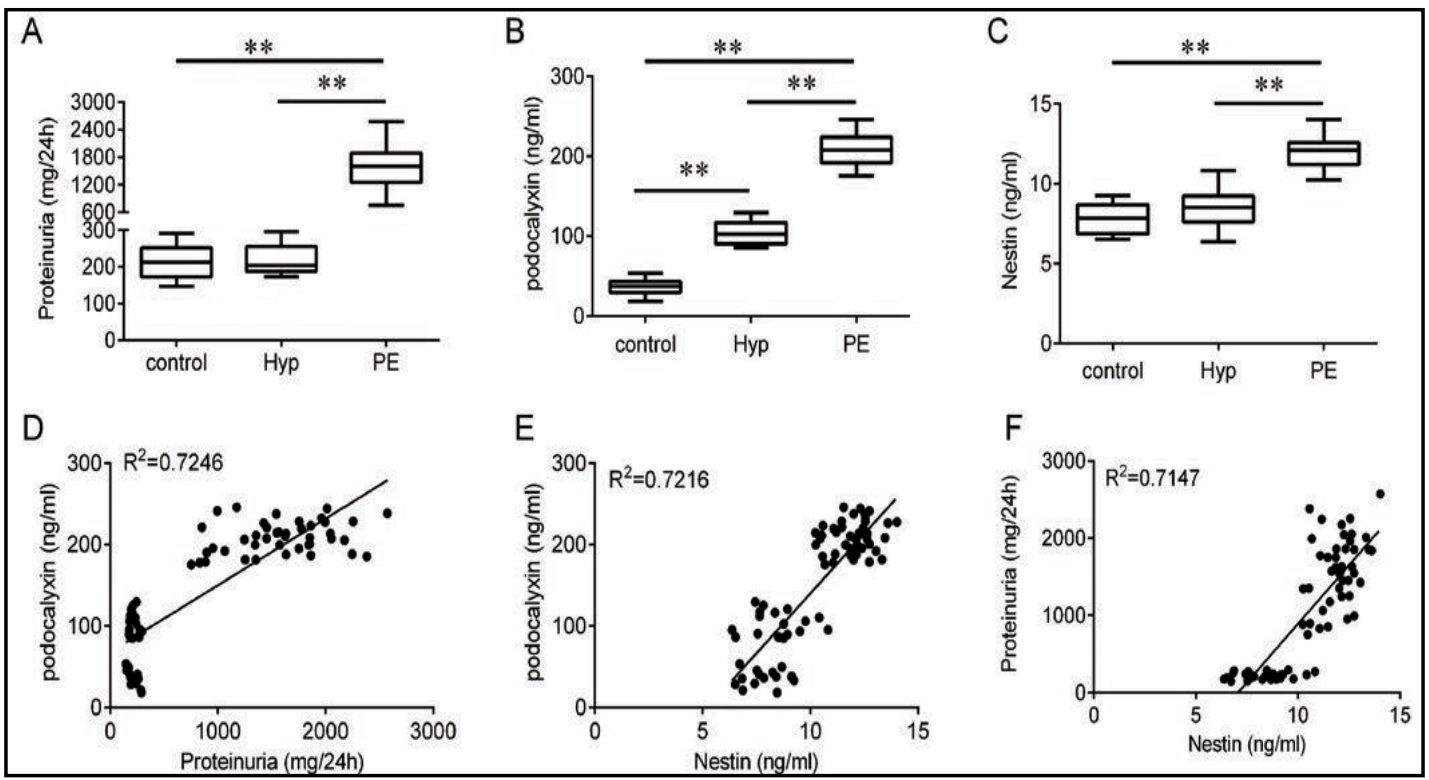

Fig. 1. Associationsamong the levels of Nestin, proteinuria and podocalyxin in urine samples. (A) The proteinuria concentration in healthysubjects, hypertensive patients and PE patients. (B) The podocalyxin concentration in urine samples from healthysubjects, hypertensive patients and PE patients. (C) The Nestin concentration in the urine samplesof healthy control, hypertensive patients and PE patients. (D) Proteinuria levels were positively correlated with the podocalyxin concentration in urine samples from PE patients. (E) Nestin levels were positively correlated with the podocalyxin concentration in urine samples from PE patients. (F) Nestin levels were positively correlated with the proteinuria concentration in urine samples from PE patients. Control, healthy subjects; Hyp, hypertensive patients; PE, preeclampsia.

Fig. 2. Ang II treatment induces Nestin down-regulation in a time- and dose dependent manner. (A) Western blotting for Podocin and Nestin after Ang II treatment at indicated time points and doses. (B) Immunofluorescence staining for F-actin (Green) and Podocin (Red) after 24 and $48 \mathrm{~h}$ of Ang II treatment. The cells were counterstained with DAPI (Blue). Magnification, 200×. (C) Flow cytometry analysis of cell apoptosis after 24 and $48 \mathrm{~h}$ of Ang II treatment. (D) Western blotting for CDK5, p35 and p53. The cells treated with saline were used as the control. ${ }^{*} \mathrm{P}<0.05$.

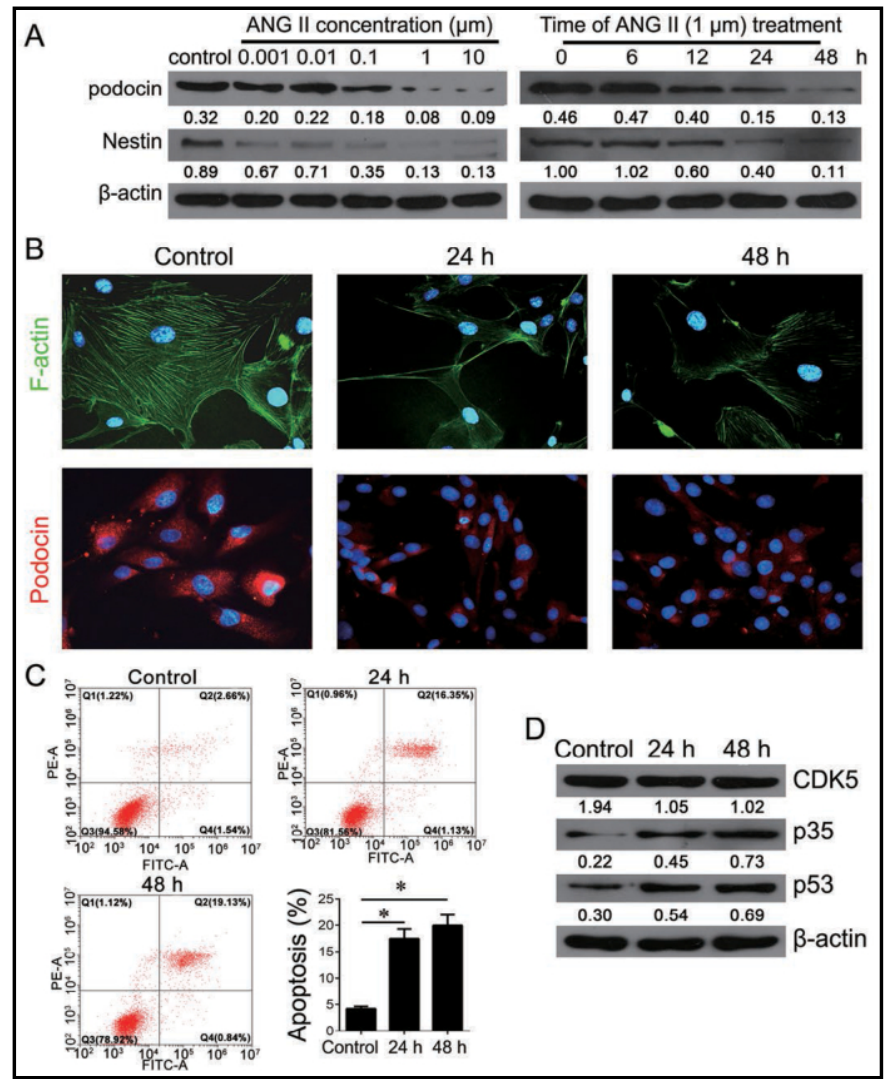




\section{Kidney Blood Pressure Research}

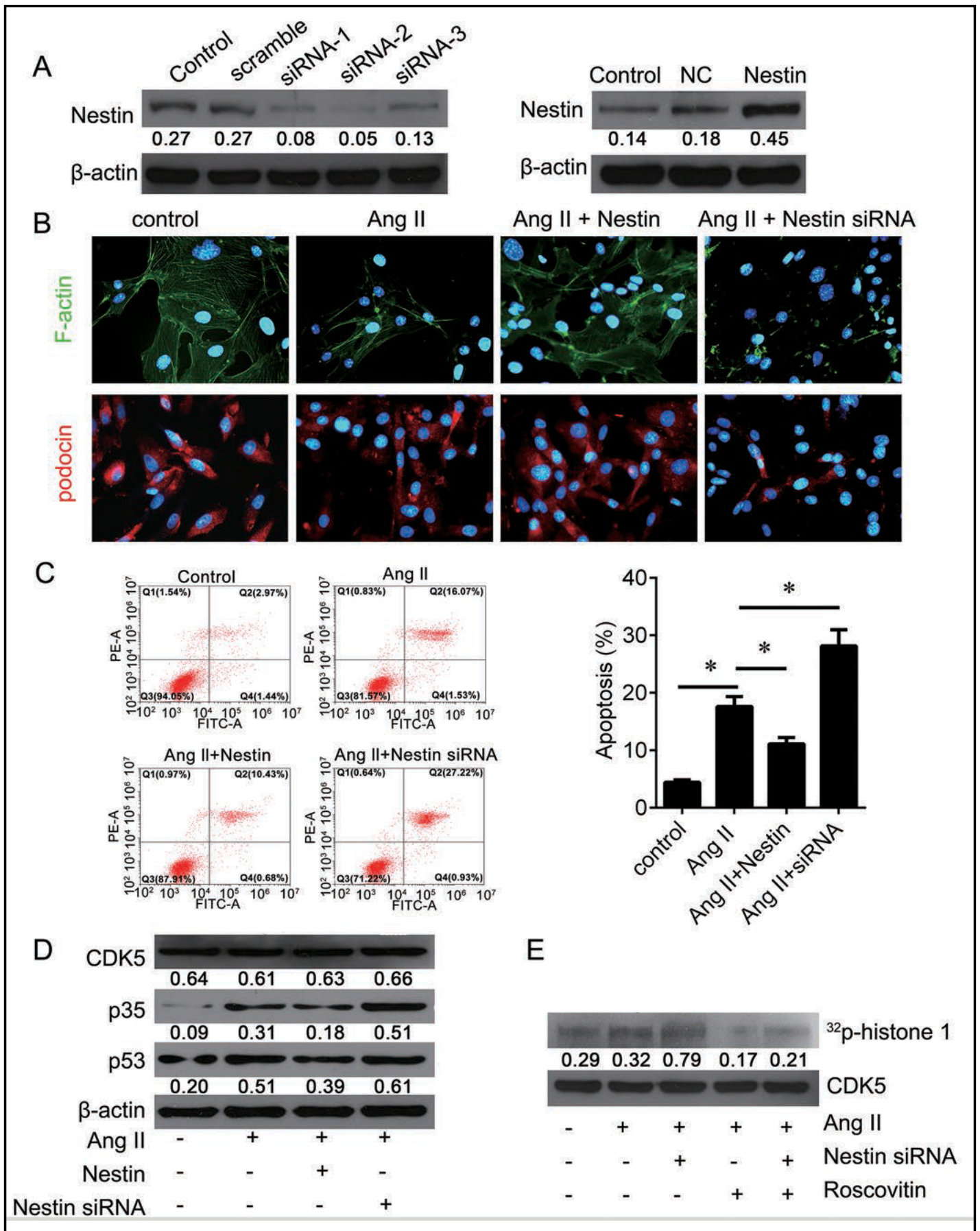

Fig. 3. Restoration of Nestin reverses Ang II induced podocyte apoptosis. (A) Western blotting for Nestin after indicated transfection. The cells treated with scramble and empty plasmid were used as the negative control (NC), while untreated cells were used as the normal control (control). (B) Immunofluorescence staining for F-actin (Green) and Podocin (Red) after indicated treatment. The cells were counterstained with DAPI (Blue). Magnification, 200×. (C) Flow cytometric analysis of cell apoptosis after indicated treatment. (D) Western blotting for CDK5, p35 and p53 after the indicated treatment. (E) Western blotting for the kiniase activity of CDK5 after the indicated treatment. ${ }^{*} \mathrm{P}<0.05$. 


\section{Kidney Blood Pressure Research}

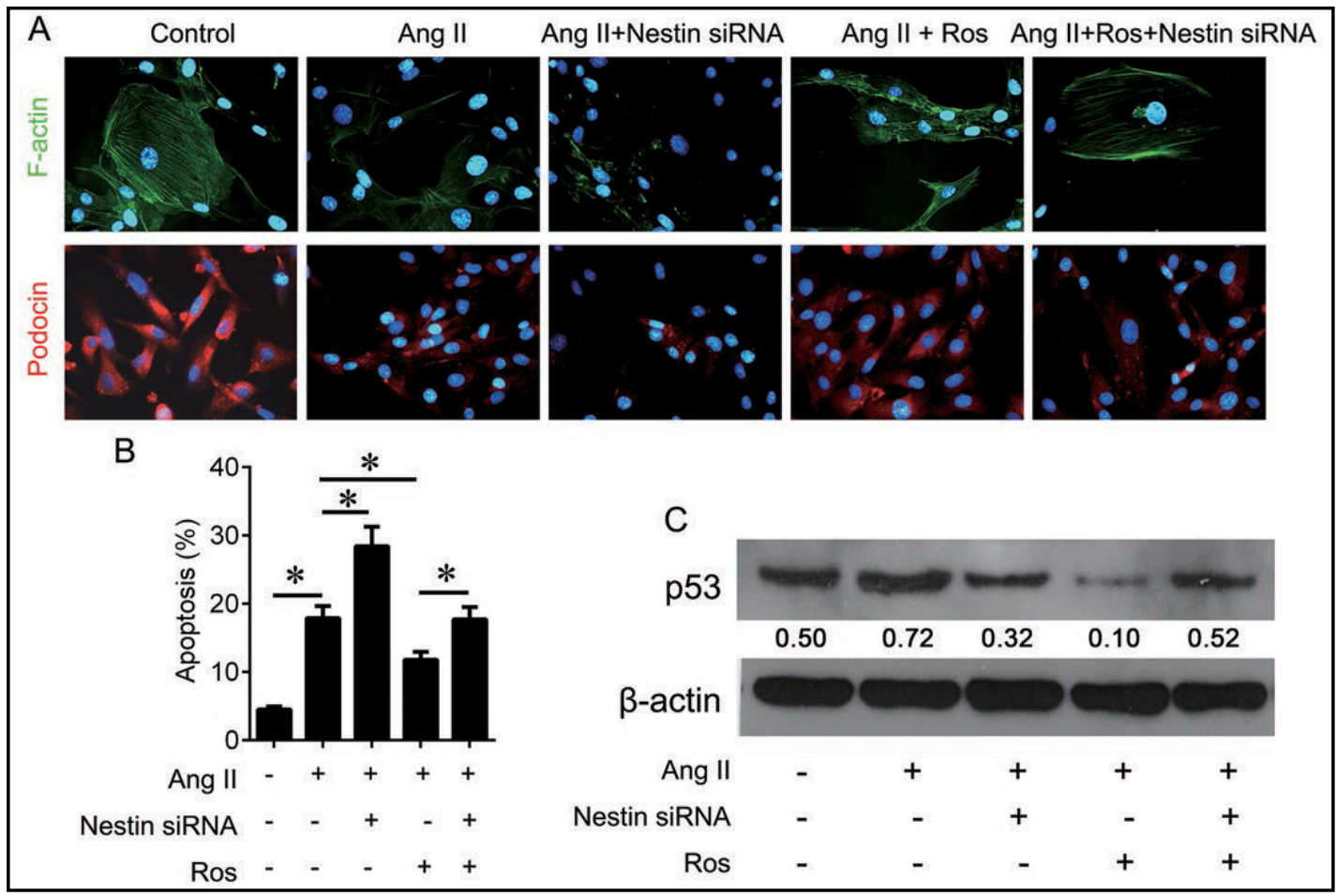

Fig. 4. CDK5 inhibition rescues Nestin knockdown-mediated podocyte apoptosis. (A) Immunofluorescence staining for F-actin (Green) and Podocin (Red) after the indicated treatment. The cells were counterstained with DAPI (Blue). Magnification, 200×. (B) Flow cytometric analysis of cell apoptosis after the indicated treatment. (C) Western blotting for p53 after the indicated treatment. ${ }^{*} \mathrm{P}<0.05$. Ros, roscovitin.

\section{Overexpression of Nestin reverses Ang II-induced podocyte apoptosis}

We examined whether restoration of Nestin level can reverse Ang II-induced podocyte apoptosis. For this purpose, we knocked down Nestin expression with small-interfering RNA (siRNA) and overexpressed Nestin by lentivirus infection in podocytes (Fig. 3A). We found that the overexpression of Nestin reversed Ang II-induced F-actin degradation and decreased Podocin expression, whereas the knockdown of Nestin exacerbated F-actin degradation and further reduced Podocin expression (Fig. 3B). As expected, rescue of the Nestin level attenuated Ang II-mediated apoptosis, while its knockdown exhibited the opposite effect (Fig. 3C). In addition, the expression of p35 and p53 was reduced in cells co-treated with Ang II and Nestin, while their expression was higher when co-treated with Ang II and Nestin siRNA than treated with Ang II alone (Fig. 3D).

However, neither the overexpression of nor the knockdown of Nestin altered the expression of CDK5 (Fig. 3D). We investigated whether Nestin regulated CDK5 kinase activity and found that Ang II treatment increased CDK5 kinase activity, and while the knockdown of Nestin had an additive effect with Ang II on CDK5 kinase activity (Fig. 3E). Moreover, cotreatment with the CDK5 kinase inhibitor Roscovitin significantly inhibited the CDK5 kinase activity induced by the knockdown of Nestin (Fig. 3E). We next tested whether the protective role of Nestin occurred via CDK5 kinase. Podocytes were treated with Ang II along with Nestin siRNA or Roscovitin or with both. The F-actin degradation and reduction of Podocin induced by Nestin siRNA were reversed by Roscovitin (Fig. 4A). The increased cell apoptosis induced by Nestin siRNA was also rescued by Roscovitin (Fig. 4B). Corresponding to cellular apoptosis, the expression of p53 was decreased by Roscovitin treatment (Fig. 4C). 


\section{Kidney Blood Pressure Research}

Restoration of Nestin improves the PE-like symptoms

We then tested the role of Nestin in PE-like animal model. The PE-like animal model was established by L-NAME administration. L-NAME injection led to significant increases in systolic pressure and proteinuria (Fig. 5A, 5B), and induced podocyte damage, which was evaluated by Podocin immunofluorescence and TUNEL assay in renal sections (Fig. 5C, 5D). L-NAME injection also resulted in an increase in p35 and p53 expression and in the reduction of Podocin and Nestin expression in renal tissue (Fig. 5E), while significantly upregulating the activity of CDK5 kinase as compared with those in the control (Fig. 5F). After being treated with Nestin, the PE animals showed lower pressure and reduced proteinuria concentration (Fig. 5A, 5B). Restoration of the Nestin level attenuated the loss of podocytes (Fig. 5C, 5D), and decreased the p35 and p53 expression and the activity of CDK5 kinase as compared with those in the control (Fig. 5E, 5F).

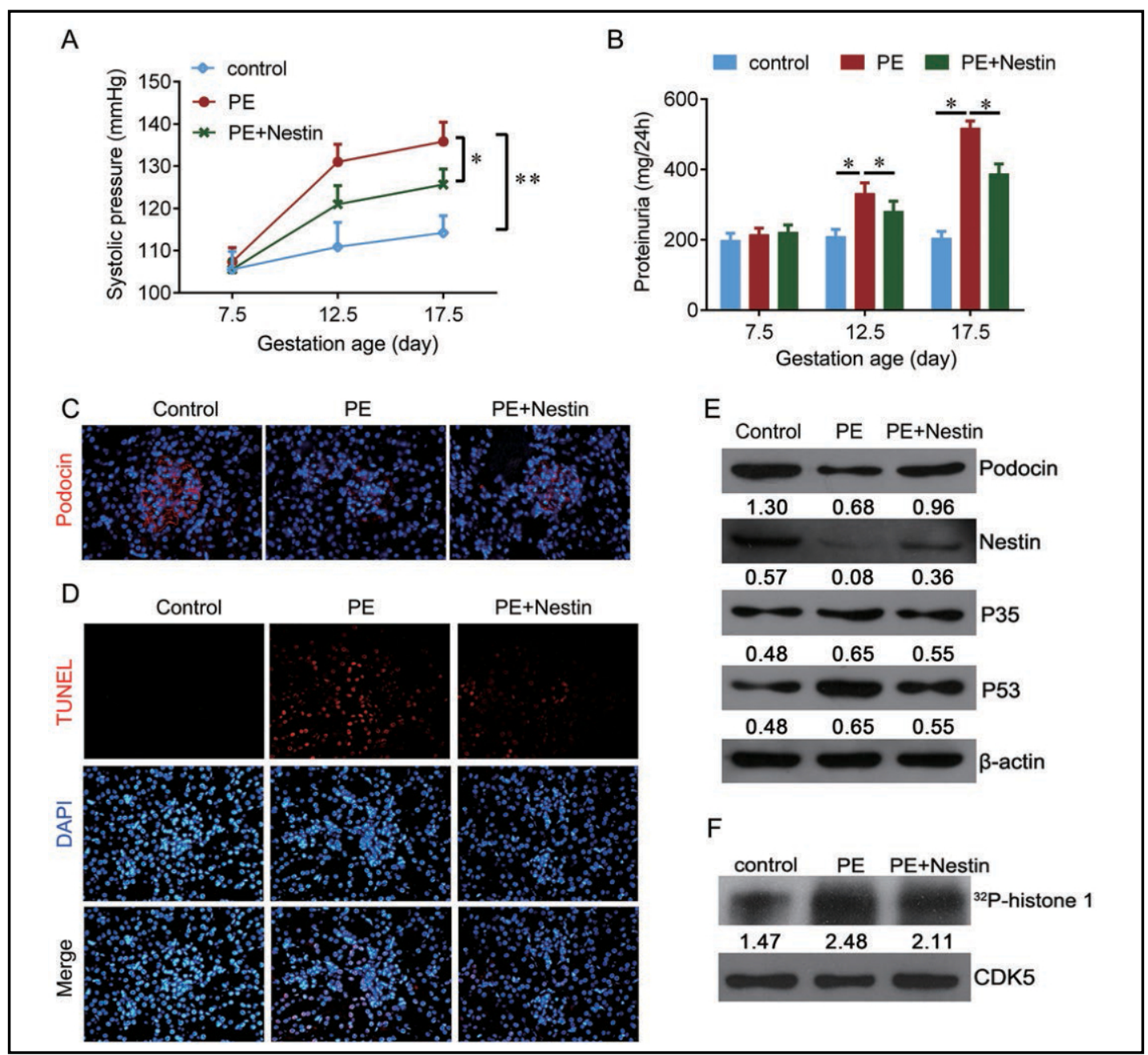

Fig. 5. Restoration of Nestin improves PE-like symptoms. (A) Systolic pressure of the tail after the indicated treatments on 7.5, 12.5 and 17.5 days of gestation. (B) The proteinuria concentration per $24 \mathrm{~h}$ after indicated treatments on 7.5, 12.5 and 17.5 days of gestation. (C) Immunofluorescence staining for Podocin (Red) in kidney sections after the indicated treatment. The sections were counterstained with DAPI (Blue). Magnification, 100×. (D) TUNEL staining (Red) in kidney sections after the indicated treatment. The sections were counterstained with DAPI (Blue). Magnification, 100×. (E) Western blotting for Podocin, Nestin, p35 and p53 in kidney tissues after the indicated treatment. (E) Western blotting for the kiniase activity of CDK5 after the indicated treatment. (F) Western blotting for the kiniase activity of CDK5 in kidney tissues after the indicated treatment. ${ }^{*} \mathrm{P}<0.05$; ${ }^{* *} \mathrm{P}<0.01$. 


\section{Kidney Blood Pressure Research}

Kidney Blood Press Res 2018;43:616-627

\begin{tabular}{l|l}
\hline DOI: $10.1159 / 000489146$ & (C) 2018 The Author(s). Published by S. Karger AG, Basel
\end{tabular}

Published onlıne: 24 April, 2018

www.karger.com/kb

625

\section{Discussion}

In this study, we found that Nestin levels were significantly higher in PE patients than in health subjects and hypertensive patients. In addition, Nestin levels were positively correlated with the level of proteinuria and Podocin. Nestin is widely expressed in a variety of human cell types during development as a neuroepithelial precursor cell marker. Under normal condition, Nestin is expressed in several glomerular cell types, including mesangial cells and endothelial cells, at the early stage of development and become confined to the podocytes in mature glomeruli $[13,14]$. Although Tomioka et al. [9] indicated that increased Nestin expression at the tubulointerstitial areas of IgA nephropathy was associated with the deterioration of the renal function, Su et al. [10] demonstrated the expression level of Nestin protein and mRNA in the kidneys of patients with IgA nephropathy and proteinuria. The authors also reported that membranous nephropathy and focal segmental glomerular sclerosis with proteinuria were significantly reduced as compared with that in normal kidney, suggesting that Nestin may play an important role in maintaining normal podocyte function. Moreover, the loss of podocytes into urine contributes to the development of glomerular diseases such as proteinuria [15]. In IgA nephropathy, podocyte injury is involved in the development of proteinuria, and the loss of podocytes predicts the progression of the proteinuria [11]. Thus, we hypothesised that the increased Nestin level in urine samples from PE patients results from the loss of podocytes in the kidney, which contributes to proteinuria.

Podocytes express intermediate filament proteins, including Vimentin, Desmin, and Nestin. Once the podocytes are injured, alterations of intermediate filament proteins may occur to enable the podocytes to undergo morphological changes on the tensile glomerular capillary wall. In the present study, we found that Ang II treatment induced F-actin degradation and led to an increase in podocyte apoptosis. Moreover, Ang II treatment decreased the expression of Nestin and Podocin in a time- and dose- dependent manner. Through gain and loss of function experiments, we demonstrated that the overexpression of Nestin level reversed Ang II-induced F-actin degradation and decreased Podocin expression. Moreover, restoration of the Nestin level attenuated the Ang II-mediated apoptosis of podocytes. Previous studies have showed that knocking down Nestin expression resulted in a remarkable derangement of the actin cytoskeleton and an increase in apoptosis in cultured podocytes $24 \mathrm{~h}$ after being incubated with puromycin aminonucleoside, while up-regulation of the Nestin expression during puromycin aminonucleoside-induced nephrosis could protect the podocytes from apoptosis, this process was mediated by maintaining the regular arrangement of the actin cytoskeleton [16]. Thus, Nestin may play a protective role for the podocytes under hypertensive conditions.

We next explored the mechanism by which Nestin protected podocytes from the apoptosis induced by Ang II and found that the knockdown of Nestin significantly increased the kinase activity of CDK5 and its activator p35. In addition, the inhibition of kinase activity of CDK5 by Roscovitin could reverse Nestin down-regulation-mediated podocyte apoptosis and actin cytoskeleton degradation. High glucose concentration could induce podocyte apoptosis and decrease the mRNA and protein expression levels of Nestin in a time-dependent manner for cultured podocytes. Moreover, the knockdown of the Nestin expression resulted in a significant increase in the high glucose-induced podocyte apoptotic rate through the regulating of CDK5 levels [17]. Liu et al. [17] also showed that the degree of albuminuria in diabetic rats was negatively correlated with the Nestin concentration and positively correlated with the CDK5 level. Roscovitine could reduce the degradation of NestinNestin and ameliorate podocyte injuries $[18,19]$. Thus, our findings indicate that the protective role of Nestin on podocytes is mediated by inhibition of the kinase activity of CDK5 when challenged by Ang II. 


\section{Kidney Blood Pressure Research}

Yang et al.: Nestin/CDK5 Improves Preeclampsia-Like Symptoms

In pulmonary hypertension, Nestin was significantly enhanced in the lungs early during the development of pulmonary hypertension, while Nestin expression returned to control levels at later time points when pulmonary hypertension became clinically evident [20]. Female mice with Nestin-ER $\alpha$ ablated in the nervous system showed greater increases in blood pressure in response to Ang II [21]. In a PE-like animal model, we confirmed that the administration of L-NAME resulted in hypertension, proteinuria and increased podocyte apoptosis in the kidney. In addition, PE could also up-regulate the kinase activity of CDK5. Nestin level restoration significantly improved these PE-like symptoms by inhibiting the kinase activity of CDK5 and by protecting the podocytes.

\section{Conclusion}

Our findings reveal that the Nestin level is increased in the urine of PE patients and that restoration of the Nestin level in podocytes could protect podocytes from apoptosis under hypertensive conditions, thereby improving PE-like symptoms. Through this study, we may find a new prognostic factor and therapy target for PE.

\section{Disclosure Statement}

The authors declare no conflict of interests.

\section{References}

1 Moussa HN, Arian SE, Sibai BM: Management of hypertensive disorders in pregnancy. Womens Health 2014;10:385-404.

-2 Hutcheon JA, Lisonkova S, Joseph KS: Epidemiology of pre-eclampsia and the other hypertensive disorders of pregnancy. Best Pract Res Clin Obstet Gynaecol 2011;25:391-403.

-3 Karumanchi SA, Granger JP: Preeclampsia and Pregnancy-Related hypertensive disorders. Hypertension 2016;67:238-242.

4 Endlich N, Endlich K: The challenge and response of podocytes to glomerular hypertension. Semin Nephrol 2012;32:327-341.

5 Muller-Deile J, Schiffer M: Renal involvement in preeclampsia: Similarities to VEGF ablation therapy. J Pregnancy 2011;2011:176973.

6 Henao DE, Saleem MA: Proteinuria in preeclampsia from a podocyte injury perspective. Curr Hypertens Rep 2013;15:600-605.

7 Tardif K, Hertig V, Duquette N, Villeneuve L, El-Hamamsy I, Tanguay JF, Calderone A: Nestin upregulation characterizes vascular remodeling secondary to hypertension in the rat. Am J Physiol Heart Circ Physiol 2015;308:H1265-H1274.

8 Hwang HS, Cho NH, Maeng YS, Kang MH, Park YW, Kim YH: Differential expression of nestin in normal and pre-eclamptic human placentas. Acta Obstet Gynecol Scand 2007;86:909-914.

\9 Tomioka M, Hiromura K, Sakairi T, Takeuchi S, Maeshima A, Kaneko Y, Kuroiwa T, Takeuchi T, Nojima Y: Nestin is a novel marker for renal tubulointerstitial injury in immunoglobulin a nephropathy. Nephrology 2010;15:568-574.

$>10$ Su W, Chen J, Yang H, You L, Xu L, Wang X, Li R, Gao L, Gu Y, Lin S, Xu H, Breyer MD, Hao CM: Expression of nestin in the podocytes of normal and diseased human kidneys. Am J Physiol Regul Integr Comp Physiol 2007;292:R1761-R1767.

11 Xu L, Yang HC, Hao CM, Lin ST, Gu Y, Ma J: Podocyte number predicts progression of proteinuria in IgA nephropathy. Mod Pathol 2010;23:1241-1250.

12 Mundel P, Reiser J, Kriz W: Induction of differentiation in cultured rat and human podocytes. J Am Soc Nephrol 1997;8:697-705. 


\section{Kidney

Yang et al.: Nestin/CDK5 Improves Preeclampsia-Like Symptoms

13 Ishizaki M, Ishiwata T, Adachi A, Tamura N, Ghazizadeh M, Kitamura H, Sugisaki Y, Yamanaka N, Naito Z, Fukuda Y: Expression of nestin in rat and human glomerular podocytes. J Submicrosc Cytol Pathol 2006;38:193-200.

14 Perry J, Ho M, Viero S, Zheng K, Jacobs R, Thorner PS: The intermediate filament nestin is highly expressed in normal human podocytes and podocytes in glomerular disease. Pediatr Dev Pathol 2007;10:369-382.

15 Sakairi T, Abe Y, Kajiyama H, Bartlett LD, Howard LV, Jat PS, Kopp JB: Conditionally immortalized human podocyte cell lines established from urine. Am J Physiol Renal Physiol 2010;298:F557-F567.

16 Wen D, You L, Zhang Q Zhang L, Gu Y, Hao CM, Chen J: Upregulation of nestin protects podocytes from apoptosis induced by puromycin aminonucleoside. Am J Nephrol 2011;34:423-434.

17 Liu W, Zhang Y, Hao J, Liu S, Liu Q, Zhao S, Shi Y, Duan H: Nestin protects mouse podocytes against high glucose-induced apoptosis by a Cdk5-dependent mechanism. J Cell Biochem 2012;113:3186-3196.

18 Liu W, Zhang Y, Liu S, Liu Q, Hao J, Shi Y, Zhao S, Duan H: The expression of intermediate filament protein nestin and its association with cyclin-dependent kinase 5 in the glomeruli of rats with diabetic nephropathy. Am J Med Sci 2013;345:470-477.

19 Bertelli E, Regoli M, Fonzi L, Occhini R, Mannucci S, Ermini L, Toti P: Nestin expression in adult and developing human kidney. J Histochem Cytochem 2007;55:411-421.

20 Saboor F, Reckmann AN, Tomczyk CU, Peters DM, Weissmann N, Kaschtanow A, Schermuly RT, Michurina TV, Enikolopov G, Muller D, Mietens A, Middendorff R: Nestin-expressing vascular wall cells drive development of pulmonary hypertension. Eur Respir J 2016;47:876-888.

-21 Xue B, Zhang Z, Beltz TG, Guo F, Hay M, Johnson AK: Genetic knockdown of estrogen receptor-alpha in the subfornical organ augments ANG II-induced hypertension in female mice. Am J Physiol Regul Integr Comp Physiol 2015;308:R507-R516. 